\title{
3-D Crank-Nicolson Finite Difference Time Domain method for dispersive media
}

\author{
Hasan Khaled Rouf ${ }^{\dagger}$, Fumie Costen ${ }^{\dagger}$, Salvador G. Garcia ${ }^{\ddagger}$ \\ ${ }^{\dagger}$ School of Electrical and Electronic Engineering, The University of Manchester, M60 1QD, U.K., \\ hasan.rouf@postgrad.manchester.ac.uk, f.costen@cs.man.ac.uk \\ ${ }^{\ddagger}$ Faculty of Sciences, University of Granada, Granada, Spain, salva@ugr.es
}

\begin{abstract}
In this paper we extend the unconditionally stable Crank-Nicolson Finite Difference Time Domain (CN-FDTD) method, to incorporate frequency-dependent media in three dimensions. A Gaussian-elimination-based direct sparse solver is used to deal with the large sparse matrix system arising from the formulation. Numerical results validate and confirm that the scheme is unconditionally stable for time steps over the CourantFriedrich-Lewy limit of classical FDTD.
\end{abstract}

Index Terms-Crank-Nicolson method, FDTD, Debye media, sparse matrix solver

\section{INTRODUCTION}

Finite Difference Time Domain (FDTD) methods produce a time domain analysis in various kinds of media using a minimal set of assumptions compared to other techniques. They are said to be the most straightforward, robust and widely applicable electromagnetic modelling techniques. Many current and emerging technological applications involve electromagnetic wave interactions with materials having frequencydispersive dielectric properties [1] necessitating modification of the classical Yee-FDTD scheme [2]. Frequency dependency has been incorporated in FDTD using several approaches: the auxiliary differential equation method, the z-transform method and the discrete convolution method.

A main drawback of the conventional FDTD method is its reduced computational efficiency resulting from the upper limit on the time step that needs to satisfy the CourantFriedrich-Lewy (CFL) stability condition [1]. Thus, when very small voxels relative to the wavelength of interest are employed to accurately model the fine geometrical details of a given geometry, an unnecessarily small time step is enforced, with an increase of the total CPU time.

An alternative to the explicit FDTD is provided by the Crank-Nicolson FDTD (CN-FDTD) method [3], which presents unconditional stability beyond CFL limit. Both methods share in common the discretization of the time and space derivatives by 2 nd order centred differences, with the only difference being that the fields affected by the curl operator are averaged in time by the CN-FDTD method, whereas in Yee-FDTD are not. The resulting scheme is a fully implicit marching-on-in-time algorithm with the same potential of the classical FDTD. However, despite its high accuracy and low anisotropy [4] it has not been widely used in time domain electromagnetics as it involves the inversion of huge sparse matrices. Instead, there have been many works attempting to simplify or approximate its implementation. However such approximations suffer up to some extent of numerical errors, which may become severe for some practical applications [5].

With the massive advancement of the technology of memory and computational resources, handling huge sparse matrices is no longer a bottleneck. This, together with the extensive researches during last two decades that resulted in highly sophisticated, robust, efficient and economical sparse solvers, makes CN-FDTD a promising affordable alternative to the classical FDTD.

In this paper we propose a new three-dimensional frequency dependent CN-FDTD method (FD-CN-FDTD). We incorporate the frequency dependence of single-pole Debye materials into CN-FDTD by means of an auxiliary differential formulation [6]. The scheme results in a sparse system of linear equations involving the three components of the electric field. A sparse direct algorithm is next used to perform the coefficient matrix decomposition, finally leading to an marchingon-in-time unconditionally stable advancing algorithm. The scheme is validated by simple numerical experiments.

\section{Fundamentals OF FD-CN-FDTD}

Maxwell's curl equations in material independent form are

$$
\begin{gathered}
\nabla \times \boldsymbol{E}=-\frac{\partial \boldsymbol{B}}{\partial t} \\
\nabla \times \boldsymbol{H}=\frac{\partial \boldsymbol{D}}{\partial t}
\end{gathered}
$$

where $\boldsymbol{E}, \boldsymbol{H}, \boldsymbol{D}$ and $\boldsymbol{B}$ are electric field, magnetic field, electric flux density and magnetic flux density respectively.

The constitutive relationships for isotropic, linear, nonmagnetic, single-pole Debye electrically-dispersive media, are in frequency domain

$$
\begin{gathered}
\boldsymbol{B}=\mu_{0} \boldsymbol{H} \\
\boldsymbol{D}=\epsilon_{0}\left(\epsilon_{\infty}+\frac{\epsilon_{\mathrm{S}}-\epsilon_{\infty}}{1+\jmath \omega \tau_{\mathrm{D}}}-\jmath \frac{\sigma}{\omega \epsilon_{0}}\right) \boldsymbol{E}
\end{gathered}
$$

where $\epsilon_{0}$ and $\mu_{0}$ are the free-space permittivity and permeability, $\epsilon_{\mathrm{S}}$ is the static permittivity, $\epsilon_{\infty}$ is the optical permittivity, $\tau_{\mathrm{D}}$ is the relaxation time and $\sigma$ is the conductivity.

Eq.(4) can be re-written as

$$
\begin{array}{r}
(\jmath \omega)^{2} \tau_{\mathrm{D}} \boldsymbol{D}+\jmath \omega \boldsymbol{D} \\
=(\jmath \omega)^{2} \epsilon_{0} \epsilon_{\infty} \tau_{\mathrm{D}} \boldsymbol{E}+\jmath \omega\left(\epsilon_{0} \epsilon_{\mathrm{S}}+\sigma \tau_{\mathrm{D}}\right) \boldsymbol{E}+\sigma \boldsymbol{E}
\end{array}
$$


By mapping $(\jmath \omega)^{m}$, in frequency domain, into $\frac{\partial^{m}}{\partial t^{m}}$, in time domain, Eq. (5) can be written as

$\tau_{\mathrm{D}} \frac{\partial^{2} \boldsymbol{D}}{\partial t^{2}}+\frac{\partial \boldsymbol{D}}{\partial t}=\epsilon_{0} \epsilon_{\infty} \tau_{\mathrm{D}} \frac{\partial^{2} \boldsymbol{E}}{\partial t^{2}}+\left(\epsilon_{0} \epsilon_{\mathrm{S}}+\sigma \tau_{\mathrm{D}}\right) \frac{\partial \boldsymbol{E}}{\partial t}+\sigma \boldsymbol{E}$

Application of Crank-Nicolson method to Eq.(1), Eq.(2), Eq.(3) and Eq. (6) and manipulation of the resultant discretised equations yield an equation only with electric field $E^{n+1}(i, j, k)$ terms:

$$
\begin{aligned}
& E_{x}{ }^{n+1}- \frac{\xi_{1}}{\xi_{4}}\left(\frac{\Delta t}{2}\right)^{2} \frac{1}{\mu} \frac{\partial^{2} E_{x}{ }^{n+1}}{\partial y^{2}}+\frac{\xi_{1}}{\xi_{4}}\left(\frac{\Delta t}{2}\right)^{2} \frac{1}{\mu} \frac{\partial^{2} E_{y}{ }^{n+1}}{\partial x \partial y} \\
&+ \frac{\xi_{1}}{\xi_{4}}\left(\frac{\Delta t}{2}\right)^{2} \frac{1}{\mu} \frac{\partial^{2} E_{z}{ }^{n+1}}{\partial z \partial x}-\frac{\xi_{1}}{\xi_{4}}\left(\frac{\Delta t}{2}\right)^{2} \frac{1}{\mu} \frac{\partial^{2} E_{x}{ }^{n+1}}{\partial z^{2}} \\
&= \frac{\xi_{1}}{\xi_{4}} D_{x}{ }^{n}+\frac{\xi_{1}}{\xi_{4}} \frac{\Delta t}{2} \frac{\partial H_{z}{ }^{n}}{\partial y}+\frac{\xi_{1}}{\xi_{4}}\left(\frac{\Delta t}{2}\right)^{2} \frac{1}{\mu} \frac{\partial^{2} E_{x}{ }^{n}}{\partial y^{2}} \\
&-\frac{\xi_{1}}{\xi_{4}}\left(\frac{\Delta t}{2}\right)^{2} \frac{1}{\mu} \frac{\partial^{2} E_{y}{ }^{n}}{\partial x \partial y}-\frac{\xi_{1}}{\xi_{4}} \frac{\Delta t}{2} \frac{\partial H_{y}{ }^{n}}{\partial z}-\frac{\xi_{1}}{\xi_{4}}\left(\frac{\Delta t}{2}\right)^{2} \frac{1}{\mu} \frac{\partial^{2} E_{z}{ }^{n}}{\partial z \partial x} \\
&+\frac{\xi_{1}}{\xi_{4}}\left(\frac{\Delta t}{2}\right)^{2} \frac{1}{\mu} \frac{\partial^{2} E_{x}{ }^{n}}{\partial z^{2}}+\frac{\xi_{1}}{\xi_{4}}\left(\frac{\Delta t}{2}\right)^{2} \frac{\partial H_{z}{ }^{n}}{\partial y}-\frac{\xi_{1}}{\xi_{4}}\left(\frac{\Delta t}{2}\right)^{2} \frac{\partial H_{y}{ }^{n}}{\partial z} \\
&+\frac{\xi_{2}}{\xi_{4}} D_{x}{ }^{n}+\frac{\xi_{3}}{\xi_{4}} D_{x}{ }^{n-1}-\frac{\xi_{5}}{\xi_{4}} E_{x}{ }^{n}-\frac{\xi_{6}}{\xi_{4}} E_{x}{ }^{n-1}
\end{aligned}
$$

$$
\begin{aligned}
& \text { efficient than the iterative methods when large number of } \\
& \text { iterations are needed, since at each time step only forward } \\
& \text { and backward solutions are required. }
\end{aligned}
$$

\section{Numerical Results}

To validate FD-CN-FDTD numerically, a computational region of size $(60 \times 60 \times 60)$ cells was considered. Half of it was filled with medium $1\left(\epsilon_{\mathrm{S}}=71.66, \epsilon_{\infty}=34.58, \sigma=0.49 \mathrm{~S} / \mathrm{m}\right.$ and $\left.\tau_{\mathrm{D}}=5.65 \mathrm{ps}\right)$ and the other half with medium 2 $\left(\epsilon_{\mathrm{S}}=87.34, \epsilon_{\infty}=49.13, \sigma=0.69 \mathrm{~S} / \mathrm{m}\right.$ and $\left.\tau_{\mathrm{D}}=26.89 \mathrm{ps}\right)$ as shown in Figure 1 A z-directed point-source was placed at $(20,30,30)$ in medium 1 , with a time evolution of a modulated Gaussian pulse centred at $3 \mathrm{GHz}$. Signals were recorded 20 cells away at $(40,30,30)$ in medium 2 . A uniform spatial sampling was taken $\left(\Delta x=\Delta y=\Delta z=10^{-3} \mathrm{~m}\right)$. two E-field equations. By applying them to each Yee-grid position, a system of linear equations of $\boldsymbol{A N}=\boldsymbol{C}$ is found, with $\boldsymbol{A}$ being extremely large and highly sparse coefficient matrix. The size of $\boldsymbol{A}$ is $\left(3 \cdot\left(N_{x}-1\right) \cdot\left(N_{y}-1\right) \cdot\left(N_{z}-1\right)\right) \times$ $\left(3 \cdot\left(N_{x}-1\right) \cdot\left(N_{y}-1\right) \cdot\left(N_{z}-1\right)\right)$ where $N_{x}, N_{y}, N_{z}$ are the size of the FDTD space in $x, y$ and $z$ directions, respectively ( $i=i_{\text {min }}, j=j_{\text {min }}$ and $k=k_{\text {min }}$ are excluded because at these points boundary conditions will be used). $N$ represents a vector with the electric field components to be solved, and $C$ is the excitation vector.

The solution of the system of equations to find the electric field is the core of the scheme. All the remaining field quantities are found in an explicit manner from the electric field. In order to provide a convenient and straightforward algorithm, Mur first order boundary conditions [7] were employed into FD-CN-FDTD.

\section{SOlution OF THE SPARSE SYSTEM}

The sparse system $\boldsymbol{A N}=\boldsymbol{C}$ can be solved by direct or iterative methods. Despite their intrinsic appeal for very large linear systems, iterative solvers are not robust compared to direct solvers [8] and often require preconditioning to improve their efficiency and robustness. As the purpose of this Letter is to verify the validity of the proposed FD-CN-FDTD, not the improvement of the way to solve $A N=C$ a direct solver approach was used. Direct solvers are robust, reliable and their latest implementations are quite memory efficient, and have efficient reordering techniques, which improve the performance to a great extent. In this paper we will use a version of sparse Gaussian elimination, which is described as the state-of-the-art in direct methods in [9]. This method chooses a pivot sequence to decompose $\boldsymbol{A}$ into LU factors,
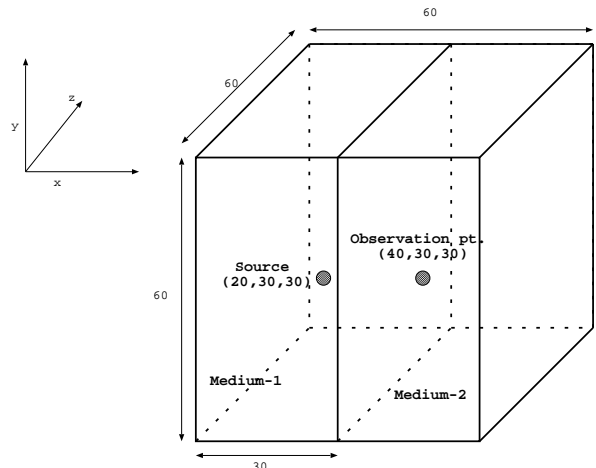

Medium 1 $\epsilon_{S}=71.66, \epsilon_{\infty}=34.58$, $\tau=5.65 \mathrm{ps}, \sigma=0.49 \mathrm{~S} / \mathrm{m}$

Medium 2: $\epsilon_{S}=87.34, \epsilon_{\infty}=49.13$,

Fig. 1. FDTD problem space for simulation with FD-CN-FDTD

As a reference, an identical setup was taken for the standard explicit frequency dependent (FD-)FDTD. The first 1200 time steps of the $E_{z}$ field component at the observation point are shown in Figure 2, computed both with FD-FDTD with $C F L N \equiv \Delta t / \Delta t_{C F L}=1$, and with FD-CN-FDTD with $C F L N=1,3,5$, where $\Delta t_{C F L}$ denotes the maximum time step allowed by the CFL stability condition. Good agreement between the signals from the proposed scheme and explicit FD-FDTD was observed. The scheme is unconditionally stable beyond CFL limit even when $C F L N$ is set to as large as 20. Numerical error increasingly appears with higher $C F L N$. Thus $\Delta t$ is no longer restricted by CFL limit but by numerical error - a characteristic possessed by other implicit schemes like ADI-FDTD [10]. CN schemes lead to finite spurious oscillations not connected with roundoff but with the nonsmooth initial conditions - this problem was also recognized by Crank and Nicolson [3] as well as reported in the standard $\tau=26.89 \mathrm{ps}, \sigma=0.69 \mathrm{~S} / \mathrm{m}$ 


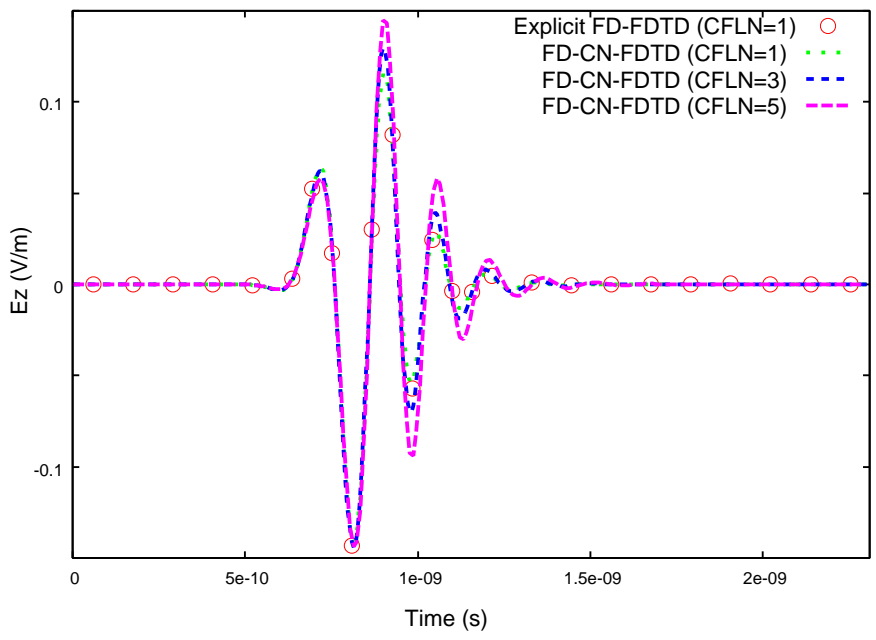

Fig. 2. The observations from both explicit scheme and Crank-Nicolson scheme

texts like [11] [12]. The reason behind the small discrepancy at higher $C F L N$ and trailing oscillations, that is seen when FD-CN-FDTD is run with even higher $C F L N$ (around 10 or more), is such characteristics inherent to the $\mathrm{CN}$ scheme.

For a $(30 \times 30 \times 30)$ cells FDTD space having environment like Figure 1 (dimensions are halved) CPU time required for LU decomposition was 633 minutes and average CPU time per iteration was 6.489 seconds when $C F L N=1$ on dual AMD Opteron 280 with $8 \mathrm{~GB}$ of memory. When the FDTD space was filled with homogeneous material of media 1 or when $C F L N>1$ there were no significant differences in these values.

\section{CONCLUSION}

A method to incorporate frequency dependent Debyedispersive media into the unconditionally stable CrankNicolson FDTD method has been presented. The proposed scheme uses a direct sparse solver, which performs the LU factorization only once (before the time-stepping begins). The major amount of the computation time is employed at this stage, keeping the computational times during time-marching similar to those of FDTD. The application of iterative methods to solve the sparse system is currently under investigation.

\section{REFERENCES}

[1] A. Taflove and S. Hagness, Computational Electromagnetics. The finitedifference Time-domain method. Boston, MA: Artech House, 2000.

[2] K. S. Yee, "Numerical solution of initial boundary value problems involving maxwell's equations in isotropic media," IEEE Trans. Antennas Propagat., vol. AP-14, 1966.

[3] J. Crank and P. Nicolson, "A practical method for numerical evaluation of solutions of partial differential equations of the heat-conduction type," Proc. Camb. Phil. Soc., vol. 43, pp. 50-67, 1947.

[4] E. L. Tan, "Efficient algorithms for crank nicolson based finite difference time domain methods," IEEE Transactions on Microwave Theory and Techniques, vol. 56(2), pp. 408-413, February, 2008.

[5] S. G. Garcia, R. G. Rubio, A. R. Bretones, and R. G. Martin, "On the dispersion relation of ADI-FDTD," IEEE MICROWAVE AND WIRELESS COMPONENTS LETTERS, vol. 16, pp. 354-356, June 2006.

[6] R. M. Joseph, S. C. Hagness, and A. Taflove, "Direct time integration of Maxwell's equations in linear dispersive media with absorption for scattering and propagation of femtosecond electromagnetic pulses," Optics Letters, vol. 16, no. 18, pp. 1412-1414, 1991.
[7] G. Mur, "Absorbing boundary conditions for the finite-difference approximation of the time-domain electromagnetic-field equations," IEEE Trans. Electromagn. Compat., vol. 23, pp. 377-382, 1981.

[8] Y. Saad, Iterative Methods for Linear Systems, p. 297. PWS Publishing, 1996.

[9] S. Salvini and G. Shaw, An evaluation of new NAG Library solvers for large sparse unsymmetric linear systems. Numerical Algorithms Group, 1996.

[10] Salvador G. Garcia, T.-W. Lee, and S. C. Hagness, "On the accuracy of the ADI-FDTD method," Antennas and Wireless Propagation Letters, vol. 1, no. 1, pp. 31-34, 2002.

[11] G. Smith, Numerical Solution of Partial Differential Equations: Finite Difference Methods. Oxford University Press, 1985.

[12] J. Strikwerda, Finite Difference Schemes and Partial Differential Equations. CRC Press Inc, Aug 1995. 